\title{
Relative Nonlinear Measure Method to Exponential Stability of Impulsive Delayed Differential Equations
}

\author{
Xueli Song, ${ }^{1,2}$ Xing Xin, ${ }^{3}$ Huiya Dai, ${ }^{2,4}$ and Jigen Peng ${ }^{2}$ \\ ${ }^{1}$ Department of Mathematics and Information Science, Chang'an University, Xian 710064, China \\ ${ }^{2}$ School of Mathematics and Statistics, Xian Jiaotong University, Xian 710049, China \\ ${ }^{3}$ The 41st Institute of the Fourth Academy of CASC, Xi'an 710025, China \\ ${ }^{4}$ School of Science, Henan University of Technology, Zhengzhou 45000, China
}

Correspondence should be addressed to Xueli Song; xlsung@chd.edu.cn

Received 17 February 2013; Revised 11 August 2013; Accepted 14 August 2013

Academic Editor: Abdelaziz Rhandi

Copyright (C) 2013 Xueli Song et al. This is an open access article distributed under the Creative Commons Attribution License, which permits unrestricted use, distribution, and reproduction in any medium, provided the original work is properly cited.

\begin{abstract}
This paper is devoted to providing a novel method to global exponential stability of impulsive delayed differential equations. By utilizing relative nonlinear measure method, several global exponential stability criteria are presented for the impulsive delayed differential equations. Compared with the Razumikhin technique and Lyapunov function method, our method is less conservative and gives a convergence rate, and one of our stability criteria is more flexible by incorporating an adjustable matrix. An example and its simulation are provided to illustrate that our method is efficient and our results are new and correct.
\end{abstract}

\section{Introduction}

Impulsive delayed differential equations are a kind of important mathematical models because they can reflect many real processes and phenomena appearing in biology, mechanics, medicine, electronics, economics, and so forth and have been successfully applied to these fields [1]. Exponential stability is a favorable quality for some applications, such as, the constructions and applications of artificial neural networks and the predictions of future economy [2]. Hence, exponential stability analysis of these equations has attracted interest in theoretical and practical applied fields [1,3-7], which have presented some significant stability criteria. Among these existing methods, Lyapunov-Razumikhin method is undoubtedly the most popular (see [1,3-7] and the relevant references therein). By analyzing their proofs, we find that some assumptions may be caused by Lyapunov-Razumikhin method itself. For example, the time delays are assumed to be smaller than the length of each impulsive interval [6] in order to employ Lyapunov-Razumikhin method although this assumption is proved to be unnecessary for the stability [7]. Moreover, the impulsive magnitude functions and the length of each impulsive interval are assumed to satisfy some inequality relations by means of $v_{0}$ class functions (see, conditions (iii) and (iv) of Theorem 2.1 in [7] and Theorem 3.1 in [6]), and the supremum or infimum of lengths of impulsive intervals are supposed to meet the specific relations (see, Lemma 1 and Theorem 1 in [3]). These mean that these assumptions may be unnecessary from the point of the equation itself. Moreover, these unnecessary assumptions may bring more difficulty to the construction of Lyapunov function and further reduce the application range of the derived stability criteria. Therefore, it is significant to explore a novel method to discuss exponential stability of the impulsive delayed differential equations from the point of the equations themselves.

Being a nonlinear generalization of matrix measure (or logarithmic norm) of a matrix, relative nonlinear measure is presented [8] and employed to effectively characterize exponential stability of continuous delayed differential equations $[2,8]$. Compared with the Lyapunov-Razumikhin method, relative nonlinear measure of coefficient operators of a differential equation can better and more essentially reflect the stability information of the differential equation because it does not need any additional assumptions on itself. Moreover, the relative nonlinear measure method has been 
to successfully characterize exponential stability of delayed and impulsive cellular neural networks [9]. It implies that the relative nonlinear measure method is also effective for describing exponential stability of a special kind of impulsive delayed differential equations. Motivated by this, the main aim of this paper is to utilize relative nonlinear measure to present several stability criteria for a general impulsive delayed differential equations from the viewpoint of theory.

This paper is organized as follows. In Section 2, we introduce some basic definitions and related knowledge. In Section 3, we present several criteria for exponential stability of the impulsive delayed differential equations, an illustrative example, and its simulation. Finally, conclusions are given in Section 4.

\section{Preliminaries}

Throughout this paper, the $n$-dimensional real vector space $\mathbb{R}^{n}$ is endowed with $l^{1}$-norm $\|\cdot\|_{1}$; that is, $\|x\|_{1}=\sum_{i=1}^{n}\left|x_{i}\right|$ for every $x=\left(x_{1}, x_{2}, \ldots, x_{n}\right)^{T} \in \mathbb{R}^{n}$, where the superscript " $T$ " denotes the transpose. Let $\mathbb{R}_{+}=[0, \infty), J \subset \mathbb{R}$ and define $\operatorname{PC}\left(J, \mathbb{R}^{n}\right)$ by

$$
\begin{aligned}
\operatorname{PC}\left(J, \mathbb{R}^{n}\right) \\
=\left\{x: J \longrightarrow \mathbb{R}^{n} \mid x\right. \text { is continuous } \\
\quad \text { everywhere except at the points } t=t_{k} \in J \\
\left.\quad \text { where } x\left(t_{k}^{-}\right) \text {and } x\left(t_{k}^{+}\right) \text {exist and } x\left(t_{k}\right)=x\left(t_{k}^{-}\right)\right\} .
\end{aligned}
$$

For $\tau>0$, we equip the space $\mathrm{PC}\left([-\tau, 0], \mathbb{R}^{n}\right)$ with the norm $\|\cdot\|$ defined by

$$
\|\phi\|=\sup _{-\tau \leq s \leq 0}\|\phi(s)\|_{1}, \quad \forall \phi \in \operatorname{PC}\left([-\tau, 0], \mathbb{R}^{n}\right) .
$$

In what follows, the symbol $\mathrm{PC}$ is used to denote $\mathrm{PC}\left([-\tau, 0], \mathbb{R}^{n}\right)$ and let $\mathbb{N}=\{1,2,3, \ldots\}$.

Firstly, we consider the following impulsive delayed differential equation:

$$
\begin{gathered}
\frac{d x(t)}{d t}=f\left(t, x_{t}\right), \quad t \geq t_{0}, t \neq t_{k}, \\
\Delta x\left(t_{k}\right)=I_{k}\left(t_{k}, x_{t_{k}}\right), \quad k \in \mathbb{N}, \\
x_{t_{0}}=\phi,
\end{gathered}
$$

where functions $f, I_{k}: \mathbb{R}_{+} \times \mathrm{PC} \rightarrow \mathbb{R}^{n}$ and $\phi \in \mathrm{PC}$ satisfy all necessary conditions for the global existence, uniqueness, and continuality of solutions for $t \geq t_{0}[1,10] ; 0 \leq t_{0}<t_{1}<t_{2}<$ $\cdots$ is a strictly increasing sequences such that $\lim _{k \rightarrow+\infty} t_{k}=$ $+\infty ; \Delta x\left(t_{k}\right)=x\left(t_{k}^{+}\right)-x\left(t_{k}\right), x\left(t_{k}^{+}\right)$denotes the right-hand limit of $x(t)$ at $t=t_{k} ; x_{t}, x_{t^{+}} \in$ PC are defined by $x_{t}(s)=x(t+s)$ and $x_{t^{+}}(s)=x\left(t^{+}+s\right)$ for all $s \in[-\tau, 0]$. For simplicity, we assume that $f(t, 0)=I_{k}(t, 0)=0$ for all $t \in \mathbb{R}_{+}$and $k \in \mathbb{N}$ such that (3) admits the trivial solution. Denote by $x(t)=x\left(t, t_{0}, \phi\right)$ the solution of (3) with the initial function $x_{t_{0}}=\phi$. We further assume that all solutions $x(t)$ of (3) are continuous except at $t_{k}, k \in \mathbb{N}$, at which $x(t)$ is left continuous; that is, $x\left(t_{k}\right)=$ $x\left(t_{k}^{-}\right), k \in \mathbb{N}$.

Definition 1. Let $f: \mathbb{R}_{+} \times \mathrm{PC} \rightarrow \mathbb{R}^{n}$ be a nonlinear operator. The constant

$$
\mathscr{M}(f, 0)=\sup _{t \in \mathbb{R}_{+}, x_{t} \in \mathrm{PC}, x_{t} \neq 0} \frac{\left\langle f\left(t, x_{t}\right)-f(t, 0), \operatorname{sign}(x(t))\right\rangle}{\left\|x_{t}\right\|}
$$

is called relative nonlinear measure of $f$ at $x_{t}=0$. Here $\langle\cdot, \cdot\rangle$ denotes the inner product in $\mathbb{R}^{n}$ and $\operatorname{sign}(x)=$ $\left(\operatorname{sign}\left(x_{1}\right), \operatorname{sign}\left(x_{2}\right), \ldots, \operatorname{sign}\left(x_{n}\right)\right)^{T}$ the sign vector of $x \in \mathbb{R}^{n}$, where $\operatorname{sign}(r)$ represents the sign function of $r \in \mathbb{R}$.

Definition 2. Let $f: \mathbb{R}_{+} \times \mathrm{PC} \rightarrow \mathbb{R}^{n}$ be a nonlinear operator. The constant

$$
\mathscr{L}^{p}(f, 0)=\sup _{t \in \mathbb{R}_{+}, x_{t} \in \mathrm{PC}, x_{t} \neq 0} \frac{\left\|f\left(t, x_{t}\right)-f(t, 0)\right\|_{1}}{\left\|x_{t}\right\|}
$$

is called minimal partial Lipschitz constant of $f$ with respect to $x_{t}=0$. The operator $f$ is called partially Lipschitz continuous with respect to $x_{t}=0$ if $\mathscr{L}^{p}(f, 0)<+\infty$.

Definition 3. The trivial solution of (3) is said to be globally exponentially stable if there exist constants $M \geq 1$ and $\gamma>0$ such that

$$
\|x(t)\|_{1} \leq M e^{-\gamma\left(t-t_{0}\right)}\|\phi\|
$$

holds for $t \geq t_{0}$, where $x(t)$ is the unique solution of (3) initiated from the function $\phi \in \mathrm{PC}$.

Lemma 4 (see [11]). If $a>c \geq 0$, for every nonnegative real number $b$,

$$
0=\lambda-a+c e^{\lambda b}
$$

has a unique positive solution.

Lemma 5 (see [12, Lemma D, Pages 389-390]). Let $\gamma$ and $p$ be constants with $0<p<\gamma$. Let $v(\cdot)$ be a continuous nonnegative function satisfying $v^{\prime}(t) \leq-\gamma v(t)+p\left\|v_{t}\right\|$ for $t \geq t_{0}$. Then one has

$$
v(t) \leq\left\|v_{t_{0}}\right\| e^{-\lambda\left(t-t_{0}\right)}
$$

for all $t \geq t_{0}$, where $\left\|v_{t_{0}}\right\|=\sup _{t_{0}-b \leq s \leq t_{0}}\|v(s)\|$ and $\lambda$ is the unique positive solution of

$$
\lambda=\gamma-p e^{\lambda b}
$$

Remark 6. It is well known that an impulsive delayed differential equation is considered to be a continuous differential equation coupled with a difference equation. At present, the impulsive delayed differential equation is usually studied by main means of the research methods of the continuous delayed differential equation. Consequently, we can also directly apply Lemma 5 to characterize the asymptotic 
behaviors of the continuous parts of the impulsive delayed differential equation (3).

In what follows, we will frequently utilize the following relations between inner product $\langle\cdot, \cdot\rangle$ and $l^{1}$-norm $\|\cdot\|_{1}$ in $\mathbb{R}^{n}$ :

$$
\begin{array}{r}
\|x\|_{1}=\langle x, \operatorname{sign}(x)\rangle, \quad\|x\|_{1} \geq\langle x, \operatorname{sign}(y)\rangle, \\
\forall x, y \in \mathbb{R}^{n} .
\end{array}
$$

\section{The Relative Nonlinear Measure Method}

In order to discuss the exponential stability of the impulsive delayed differential equation (3), we make the following assumption.

(H) The impulsive magnitude operator $I_{k}:\left[t_{0},+\infty\right) \times$ PC $\rightarrow \mathbb{R}^{n}$ satisfies

$$
\left\|I_{k}\left(t_{k}, x_{t_{k}}\right)\right\|_{1} \leq N\left\|x\left(t_{k}\right)\right\|_{1}
$$

for $k \in \mathbb{N}$ and some constant $N>0$.

Theorem 7. Let the assumption ( $H)$ hold. If the relative nonlinear measure of $f$ at $x_{t}=0$ is negative, that is,

$$
\mathscr{M}(f, 0)<0
$$

then the trivial solution of (3) is globally exponentially stable. That is, there exists a constant $M \geq 1$ such that the solution $x(t)$ of (3) initiated from the function $\phi \in \mathrm{PC}$ satisfies

$$
\|x(t)\|_{1} \leq M e^{-\sigma\left(t-t_{0}\right)} \cdot\|\phi\|
$$

for all $t \geq t_{0}$, where $\sigma$ is the unique positive solution of

$$
\sigma+\mathscr{M}(f, 0) e^{\sigma \tau}=0 .
$$

Proof. Let $x(t)$ be any solution of the impulsive delayed differential equation (3) with the initial function $x_{t_{0}}=\phi \epsilon$ PC. Let $t \in\left[t_{0}, t_{1}\right) \cup\left(t_{k}, t_{k+1}\right), k \in \mathbb{N}$, and from the relations (10) we derive that

$$
\frac{\|x(t)\|_{1}-\|x(t-s)\|_{1}}{s} \leq \frac{1}{s}\langle x(t)-x(\mathrm{t}-s), \operatorname{sign}(x(t))\rangle
$$

holds for all sufficiently small $s>0$ such that $x(t)$ and $x(t-s)$ simultaneously belong to some interval $\left[t_{0}, t_{1}\right)$ or $\left(t_{k_{0}}, t_{k_{0}+1}\right)$. Consequently, the function $t \mapsto\|x(t)\|_{1}$ is almost everywhere absolutely continuous in $\left[t_{0},+\infty\right)$, which implies that derivatives of $\|x(t)\|_{1}$ exist almost everywhere in $\left[t_{0},+\infty\right)$. Furthermore, along the solution trajectories of (3) we conclude that derivatives of $\left\|x(t)_{1}\right\|$ satisfy

$$
\begin{aligned}
\frac{d\|x(t)\|_{1}}{d t} & =\left\langle\frac{d x(t)}{d t}, \operatorname{sign}(x(t))\right\rangle \\
& =\left\langle f\left(t, x_{t}\right), \operatorname{sign}(x(t))\right\rangle \\
& =\left\langle f\left(t, x_{t}\right)-f(t, 0), \operatorname{sign}(x(t))\right\rangle \\
& \leq \mathscr{M}(f, 0)\left\|x_{t}\right\| .
\end{aligned}
$$

The combination of condition (12) and Lemmas 4 and 5 implies that

$$
\|x(t)\|_{1} \leq e^{-\sigma\left(t-t_{0}\right)}\|\phi\|
$$

holds for all $t \in\left[t_{0}, t_{1}\right) \cup\left(t_{k}, t_{k+1}\right), k \in \mathbb{N}$, where $\sigma$ is the unique positive solution of

$$
\sigma+\mathscr{M}(f, 0) e^{\sigma \tau}=0
$$

According to the assumption $(\mathrm{H})$ and $I_{k}\left(t_{k}, x_{t_{k}}\right)=x\left(t_{k}^{+}\right)-$ $x\left(t_{k}\right)$, we enjoy

$$
\begin{aligned}
\left\|x\left(t_{k}^{+}\right)\right\|_{1} & =\left\|x\left(t_{k}\right)+I_{k}\left(t_{k}, x_{t_{k}}\right)\right\|_{1} \\
& \leq\left\|x\left(t_{k}\right)\right\|_{1}+\left\|I_{k}\left(t_{k}, x_{t_{k}}\right)\right\|_{1} \\
& \leq(1+N)\left\|x\left(t_{k}\right)\right\|_{1}, \quad k \in \mathbb{N} .
\end{aligned}
$$

From (17) and (19) we derive

$$
\begin{aligned}
\left\|x\left(t_{k}^{+}\right)\right\|_{1} & \leq(1+N)\left\|x\left(t_{k}\right)\right\|_{1} \\
& =(1+N)\left\|x\left(t_{k}^{-}\right)\right\|_{1} \\
& \leq(1+N) e^{-\sigma\left(t-t_{0}\right)}\|\phi\|, \quad k \in \mathbb{N} .
\end{aligned}
$$

Taking $M=1+N$, we can easily derive that (13) holds for all $t \geq t_{0}$.

Remark 8. From the point of impulsive stabilization of unstable delayed differential equations, papers $[6,7]$ have discussed (3) by Lyapunov-Razumikhin method and have derived some significant stabilization conditions. In order to utilize Lyapunov-Razumikhin method, paper [6] assumed the time delays to be smaller than the length of each impulsive interval. Moreover, both of papers [6,7] required the impulsive magnitude operator $I_{k}$ and the length of each impulsive interval to satisfy special inequality relations by means of $v_{0}$ class functions (see, conditions (iii) and (iv) of Theorem 2.1 in [7] and Theorem 3.1 in [6]). However, our method does not need these assumptions. Compared with results in $[6,7]$, hence, our results are new and may even be improvements of their results from the point of verifying the exponential stability of impulsive delayed differential equations.

Remark 9. Paper [3] has studied exponential stability of the impulsive delayed differential equation (3) by LyapunovRazumikhin method and has presented several significant stability criteria with the assumption on the supremum or infimum of lengths of impulsive intervals satisfying special relations. Our method is not dependent on the assumption. Compared with results in [3], our result is new.

Equation (3) enjoys a general form of impulsive functional differential equations. The functional $f$ sometimes acts 
on two separate parts: undelayed state $x(t)$ and delayed one $x_{t}$; that is,

$$
\begin{gathered}
\frac{d x(t)}{d t}=F(t, x(t))+G\left(t, x_{t}\right), \quad t \neq t_{k}, t \geq t_{0}, \\
\Delta x\left(t_{k}\right)=I_{k}\left(t_{k}, x_{t_{k}}\right), \quad k \in \mathbb{N}, \\
x_{t_{0}}=\phi,
\end{gathered}
$$

where the nonlinear coefficient operators $F$ and $G$ satisfy assumptions of $[1,10]$ on their respective domains. Other assumptions of (21) are the same as ones of (3). For this special form, we can present the following corresponding stability criterion by means of the definition of the relative nonlinear measure of $F$ at $x=0 \in \mathbb{R}^{n}$.

Theorem 10. Let the assumption $(H)$ hold. $F: \mathbb{R}_{+} \times \mathbb{R}^{n} \rightarrow$ $\mathbb{R}^{n}$ is a nonlinear operator and $\mathscr{M}(F, 0)$ denotes the relative nonlinear measure of $F$ at $x=0$; that is,

$$
\begin{aligned}
& \mathscr{M}(F, 0) \\
& =\sup _{t \in \mathbb{R}_{+}, x(t) \in \mathbb{R}^{n}, x(t) \neq 0} \frac{\langle F(t, x(t))-F(t, 0), \operatorname{sign}(x(t))\rangle}{\|x(t)\|_{1}} .
\end{aligned}
$$

$G: \mathbb{R}_{+} \times \mathrm{PC} \rightarrow \mathbb{R}^{\mathrm{n}}$ is partially Lipschitz continuous with respect to $x_{t}=0$. If

$$
\mathscr{M}(F, 0)+\mathscr{L}^{p}(G, 0)<0
$$

holds, then the trivial solution of (21) is globally exponentially stable; that is, there exists a constant $M \geq 1$ such that

$$
\|x(t)\|_{1} \leq M e^{-\sigma\left(t-t_{0}\right)}\|\phi\|
$$

holds for all $t \geq t_{0}$, where $\sigma$ is the unique positive solution of

$$
0=\sigma+\mathscr{M}(F, 0)+\mathscr{L}^{p}(G, 0) \cdot e^{\sigma \tau}
$$

Proof. Proof of the theorem is similar to that of Theorem 7. In detail, let $x(t)$ be any solution of the impulsive delayed differential equation (21) with the initial function $x_{t_{0}}=\phi$. Let $t \in\left[t_{0}, t_{1}\right) \cup\left(t_{k}, t_{k+1}\right), k \in \mathbb{N}$, and from the relations (10) we derive that

$$
\frac{\|x(t)\|_{1}-\|x(t-s)\|_{1}}{s} \leq \frac{1}{s}\langle x(t)-x(t-s), \operatorname{sign}(x(t))\rangle
$$

holds for all sufficiently small $s>0$ such that $x(t)$ and $x(t-s)$ simultaneously belong to some interval $\left[t_{0}, t_{1}\right)$ or $\left(t_{k}, t_{k+1}\right)$. Consequently, the function $t \mapsto\|x(t)\|_{1}$ is almost everywhere absolutely continuous in $\left[t_{0},+\infty\right)$, which implies that derivatives of $\|x(t)\|_{1}$ exist almost everywhere in $\left[t_{0},+\infty\right)$. Furthermore, let $t \in\left[t_{0}, t_{1}\right) \cup\left(t_{k}, t_{k+1}\right), k \in \mathbb{N}$, and from (21) we conclude that derivatives of $\|x(t)\|_{1}$ satisfy

$$
\begin{aligned}
\frac{d\|x(t)\|_{1}}{d t}= & \left\langle\frac{d x(t)}{d t}, \operatorname{sign}(x(t))\right\rangle \\
= & \left\langle F(t, x(t))+G\left(t, x_{t}\right), \operatorname{sign}(x(t))\right\rangle \\
= & \langle F(t, x(t))-F(t, 0), \operatorname{sign}(x(t))\rangle \\
& +\left\langle G\left(t, x_{t}\right)-G(t, 0), \operatorname{sign}(x(t))\right\rangle \\
\leq & \langle F(t, x(t))-F(t, 0), \operatorname{sign}(x(t))\rangle \\
& +\left\|G\left(t, x_{t}\right)-G(t, 0)\right\| \\
\leq & \langle F(t, x(t))-F(t, 0), \operatorname{sign}(x(t))\rangle \\
& +\mathscr{L}^{p}(G, 0)\left\|x_{t}\right\| \\
\leq & \mathscr{M}(F, 0)\|x(t)\|_{1}+\mathscr{L}^{p}(G, 0)\left\|x_{t}\right\| .
\end{aligned}
$$

The combination of condition (23) and Lemmas 4 and 5 implies that

$$
\|x(t)\|_{1} \leq e^{-\sigma\left(t-t_{0}\right)}\|\phi\|
$$

holds for all $t \in\left[t_{0}, t_{1}\right) \cup\left(t_{k}, t_{k+1}\right), k \in \mathbb{N}$, where $\sigma$ is the unique positive solution of

$$
0=\sigma+\mathscr{M}(F, 0)+\mathscr{L}^{p}(G, 0) \cdot e^{\sigma \tau} .
$$

According to the assumption $(\mathrm{H})$, we enjoy

$$
\begin{aligned}
\left\|x\left(t_{k}^{+}\right)\right\|_{1} & =\left\|x\left(t_{k}\right)+I_{k}\left(t_{k}, x_{t_{k}}\right)\right\|_{1} \\
& \leq(1+N)\left\|x\left(t_{k}\right)\right\|_{1}, \quad k \in \mathbb{N} .
\end{aligned}
$$

From (28) and (30) we derive

$$
\begin{aligned}
\left\|x\left(t_{k}^{+}\right)\right\|_{1} & \leq\left\|(1+N) x\left(t_{k}\right)\right\|_{1} \\
& =\left\|(1+N) x\left(t_{k}^{-}\right)\right\|_{1} \\
& \leq(1+N) e^{-\sigma\left(t-t_{0}\right)}\|\phi\|, \quad k \in \mathbb{N} .
\end{aligned}
$$

Consequently, we conclude that (24) holds for all $t \geq t_{0}$ if we take $M=1+N$.

In conclusion, Theorems 7 and 10 can be applied to discuss the exponential stability of a general nonlinear impulsive delayed differential equations. Obviously, they can be used to characterize the exponential stability of the following linear impulsive delayed differential equation:

$$
\begin{gathered}
\frac{d x(t)}{d t}=A x(t)+B x(t-\tau(t)), \quad t \geq 0, t \neq t_{k}, \\
\Delta x(t)=C_{k}(x(t)), \quad t=t_{k}, k \in \mathbb{N}, \\
x_{0}=\phi,
\end{gathered}
$$

where matrices $A, B: \mathbb{R}^{n} \rightarrow \mathbb{R}^{n}$ and $C_{k}$ satisfies $(\mathrm{H}), 0 \leq$ $\tau(t) \leq \tau$. 
It is worth being noted that relative nonlinear measure of a matrix $A$ is just logarithmic norm (matrix measure) $\mu(A)$ of the matrix, and minimal partial Lipschitz constant of a matrix is just the matrix norm [13]. Based on this, combined with the technique of the adjustable matrix in [9], we can immediately derive the following result.

Corollary 11. If there exists some diagonal matrix $D=$ $\operatorname{diag}\left(d_{1}, d_{2}, \ldots, d_{n}\right)$ with $d_{i}>0$ such that the inequality

$$
\mu_{1}(A D)+\|B D\|_{1}<0
$$

holds, the trivial solution of (32) is globally exponentially stable; that is, there exists a constant $M \geq 1$ such that

$$
\|x(t)\|_{1} \leq M e^{-\sigma t}\|\phi\|
$$

holds for all $t \geq 0$, where $\sigma$ is the unique positive solution of

$$
0=\sigma \cdot \min _{1 \leq i \leq n} d_{i}+\mu_{1}(A D)+\|B D\|_{1} \cdot e^{\sigma \tau} .
$$

Here $\mu_{1}(A D)$ and $\|B D\|_{1}$ represent matrix measure of the matrix $A D$ and matrix norm of the matrix $B D$ with respect to $\|\cdot\|_{1}$, respectively.

Remark 12. According to definitions of matrix measure, matrix norm, and $\|\cdot\|_{1}$, we can easily derive

$$
\begin{gathered}
\mu_{1}(A D)=\max _{1 \leq j \leq n}\left\{d_{j} a_{j j}+\sum_{i=1, i \neq j}^{n} d_{j}\left|a_{i j}\right|\right\}, \\
\|B D\|_{1}=\max _{1 \leq j \leq n}\left\{\sum_{i=1}^{n} d_{j}\left|b_{i j}\right|\right\} .
\end{gathered}
$$

Hence, condition (33) can be changed into the following form conveniently verified:

$$
\max _{1 \leq j \leq n}\left\{d_{j} a_{j j}+\sum_{i=1, i \neq j}^{n} d_{j}\left|a_{i j}\right|\right\}+\max _{1 \leq j \leq n}\left\{\sum_{i=1}^{n} d_{j}\left|b_{i j}\right|\right\}<0 .
$$

Example 13. Consider the following linear impulsive delayed differential equation:

$$
\begin{gathered}
x^{\prime}(t)=A x(t)+B x\left(t-\frac{3}{2}(2+\sin (t))\right), \quad t \neq t_{k}, t \geq 0, \\
\Delta x(t)=C_{k} x(t), \quad t=t_{k}, k \in \mathbb{N}, \\
x_{0}=\phi,
\end{gathered}
$$

where $C_{k}=\operatorname{diag}(-2,-5,-7)$,

$$
A=\left(\begin{array}{ccc}
-2 & 3 & -1.5 \\
0 & -15 & 1 \\
0.15 & 8 & -3
\end{array}\right), \quad B=\left(\begin{array}{ccc}
0.1 & 1.2 & 0.11 \\
-0.13 & 0.15 & 0.2 \\
0.12 & -1.8 & 0.037
\end{array}\right) \text {. }
$$

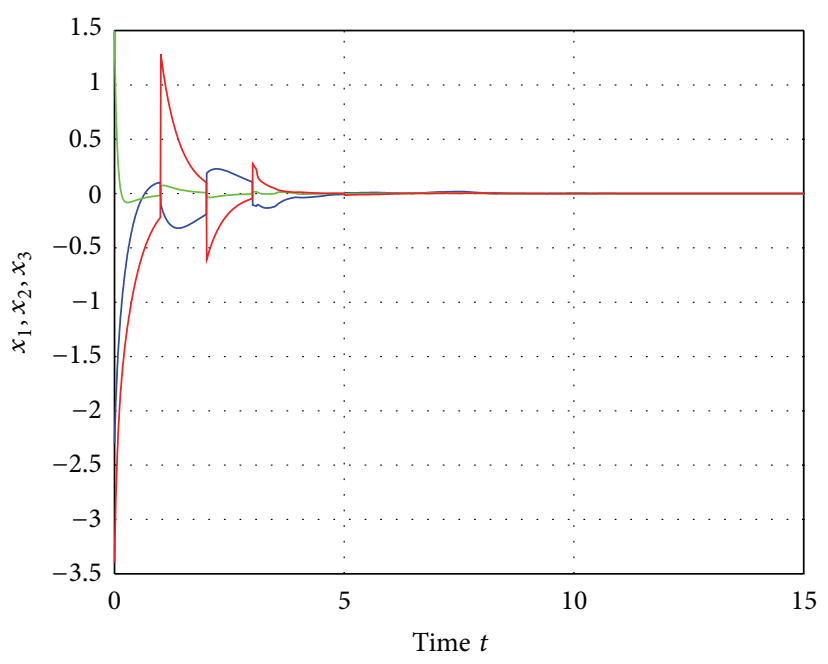

$$
\begin{array}{r}
-x_{1} \\
-x_{2} \\
-x_{3}
\end{array}
$$

FIGURE 1: Simulation of exponential convergence of (39).

Obviously, $\tau(t)=(3 / 2)(2+\sin (t)) \leq 4.5=\tau$ and $t-$ $\tau(t)=t-(3 / 2)(2+\sin (t))$ are not strictly increasing. Moreover, none of any restriction is imposed on lengths of the impulsive intervals. Consequently, the stability principles in $[3,6,7]$ are unapplicable to deal with (39). Taking $d_{1}=2, d_{2}=0.1$, and $d_{3}=1$, we can easily verify

$$
\begin{gathered}
\max _{1 \leq j \leq n}\left\{d_{j} a_{j j}+\sum_{i=1, i \neq j}^{n} d_{j}\left|a_{i j}\right|\right\}+\max _{1 \leq j \leq n}\left\{\sum_{i=1}^{n} d_{j}\left|b_{i j}\right|\right\} \\
=-0.5+0.475=-0.025<0 .
\end{gathered}
$$

From condition (38) we conclude that the trivial solution of the delayed impulsive equation (39) is globally exponentially stable with the convergence rate which is the unique solution of

$$
0.1 \sigma-0.4+0.7 e^{4.5 \sigma}=0 .
$$

The numerical simulation of this delayed impulsive equation is given in Figure 1 with respect to the initial functions:

$$
\begin{aligned}
& \phi_{1}(s)= \begin{cases}0, & s \in[-1,0), \\
-2.3, & s=0 ;\end{cases} \\
& \phi_{2}(s)= \begin{cases}0, & s \in[-1,0), \\
1.5, & s=0 ;\end{cases} \\
& \phi_{3}(s)= \begin{cases}0, & s \in[-1,0), \\
-3.4, & s=0 .\end{cases}
\end{aligned}
$$

\section{Conclusions}

The global exponential stability of the impulsive delayed differential equations has been discussed by means of the relative nonlinear measure. Several global exponential stability 
criteria have been provided for different forms of impulsive delayed differential equations. Compared with the existing methods, our method is less conservative and gives the convergence rate, and one of our stability criteria is more flexible by incorporating an adjustable matrix. An example and its simulation have been given to illustrate that our method is efficient and our results are new and correct.

\section{Acknowledgments}

This work was supported by the Natural Science Foundation of China under the Contacts nos. 11201038 and 11131006, Natural Science Foundation of Shaanxi Province under the Contact no. 2011JQ1004, and the Special Fund for Basic Scientific Research of Central Colleges under the Contact nos. 2013G2121017 and CHD2012TD015.

\section{References}

[1] I. Stamova, Stability Analysis of Impulsive Functional Differential Equations, Walter de Gruyter, New York, NY, USA, 2009.

[2] X. Song and J. Peng, "Exponential stability of equilibria of differential equations with time-dependent delay and non-Lipschitz nonlinearity," Nonlinear Analysis, vol. 11, no. 5, pp. 3628-3638, 2010.

[3] W. H. Chen and W. X. Zheng, "Exponential stability of nonlinear time-delay systems with delayed impulse effects," Automatica, vol. 47, no. 5, pp. 1075-1083, 2011.

[4] B. Liu, X. Liu, K. L. Teo, and Q. Wang, "Razumikhin-type theorems on exponential stability of impulsive delay systems," IMA Journal of Applied Mathematics, vol. 71, no. 1, pp. 47-61, 2006.

[5] Q. Wang and X. Liu, "Exponential stability for impulsive delay differential equations by Razumikhin method," Journal of Mathematical Analysis and Applications, vol. 309, no. 2, pp. 462-473, 2005.

[6] Q. Wang and X. Liu, "Impulsive stabilization of delay differential systems via the Lyapunov-Razumikhin method," Applied Mathematics Letters, vol. 20, no. 8, pp. 839-845, 2007.

[7] Q. Wu, J. Zhou, and L. Xiang, "Global exponential stability of impulsive differential equations with any time delays," Applied Mathematics Letters, vol. 23, no. 2, pp. 143-147, 2010.

[8] H. Qiao, J. Peng, and Z. B. Xu, "Nonlinear measures: a new approach to exponential stability analysis for Hopfield-type neural networks," IEEE Transactions on Neural Networks, vol. 12, no. 2, pp. 360-370, 2001.

[9] X. Song, X. Xin, and W. Huang, "Exponential stability of delayed and impulsive cellular neural networks with partially Lipschitz continuous activation functions," Neural Networks, vol. 29-30, pp. 80-90, 2012.

[10] G. Ballinger and X. Liu, "Existence and uniqueness results for impulsive delay differential equations," Dynamics of Continuous, Discrete and Impulsive Systems, vol. 5, no. 1-4, pp. 579-591, 1999.

[11] J. Peng, H. Qiao, and Z. B. Xu, "A new approach to stability of neural networks with time-varying delays," Neural Networks, vol. 15, no. 1, pp. 95-103, 2002.

[12] R. D. Driver, Ordinary and Delay Differential Equations, Springer, New York, NY, USA, 1977.

[13] T. Mori, N. Fukuma, and M. Kuwahara, "On an estimate of the decay rate for stable linear delay systems," International Journal of Control, vol. 36, no. 1, pp. 95-97, 1982. 


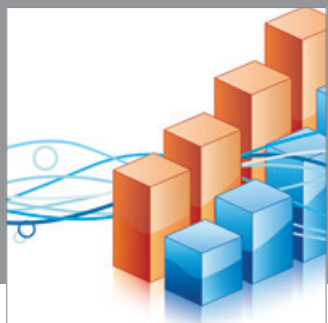

Advances in

Operations Research

mansans

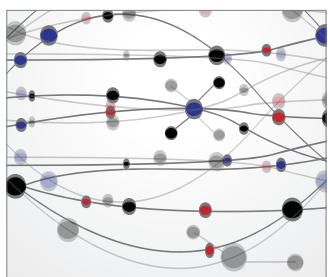

The Scientific World Journal
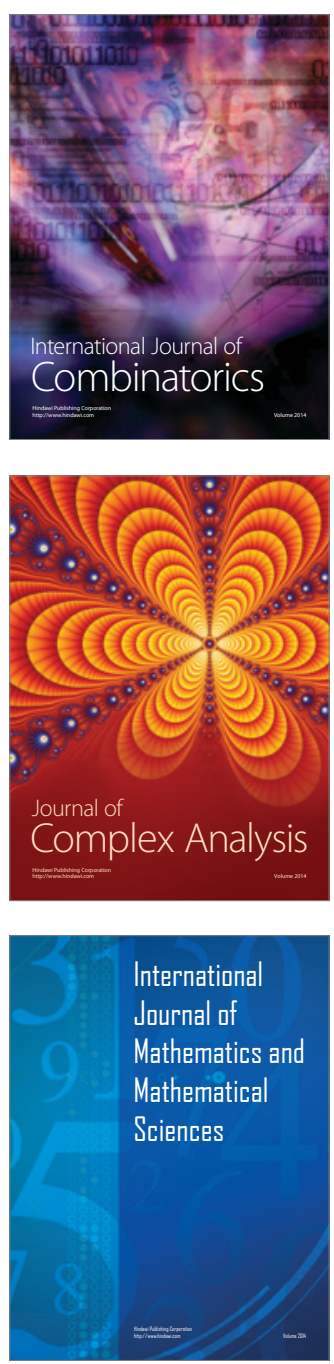
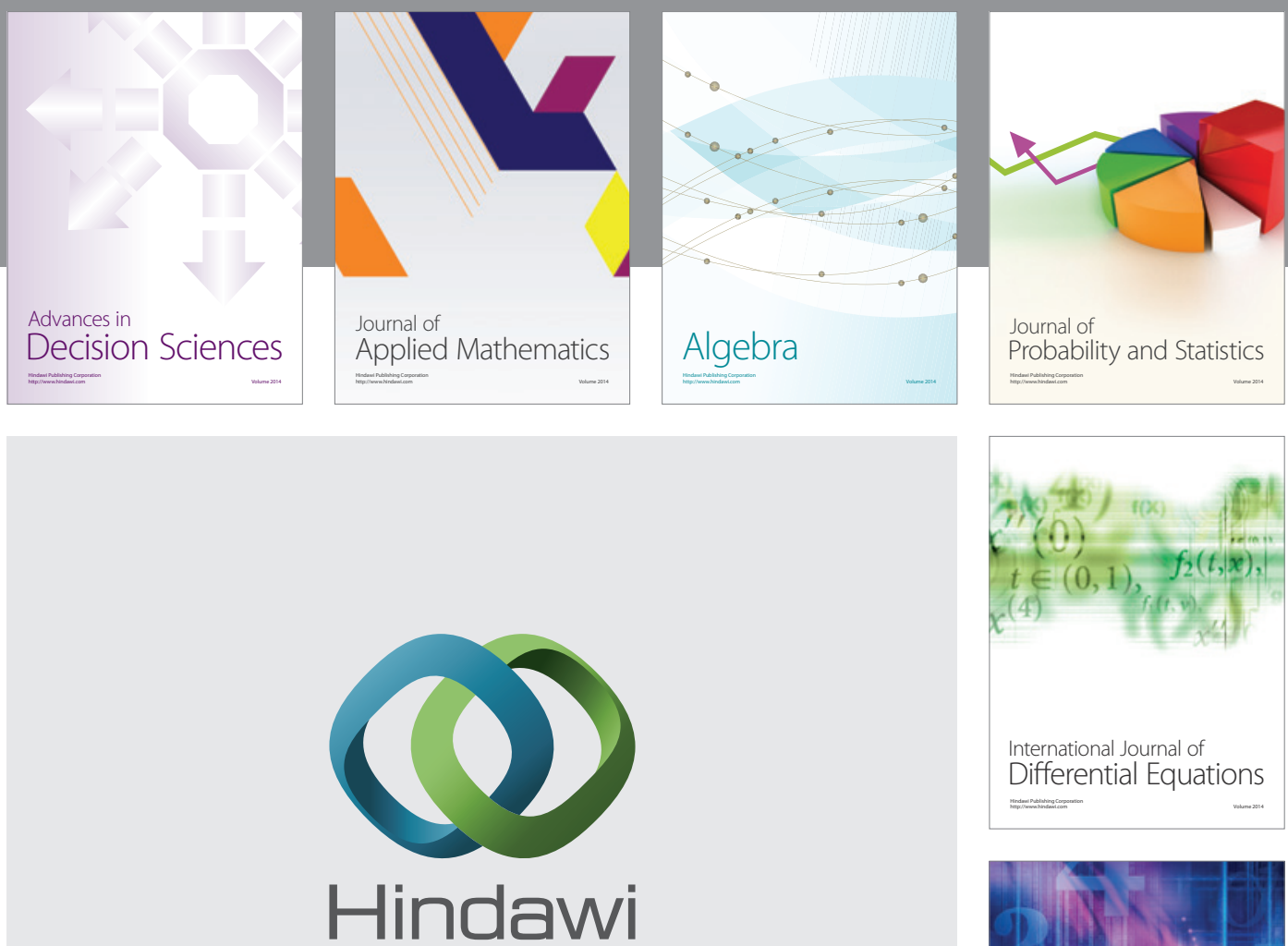

Submit your manuscripts at http://www.hindawi.com
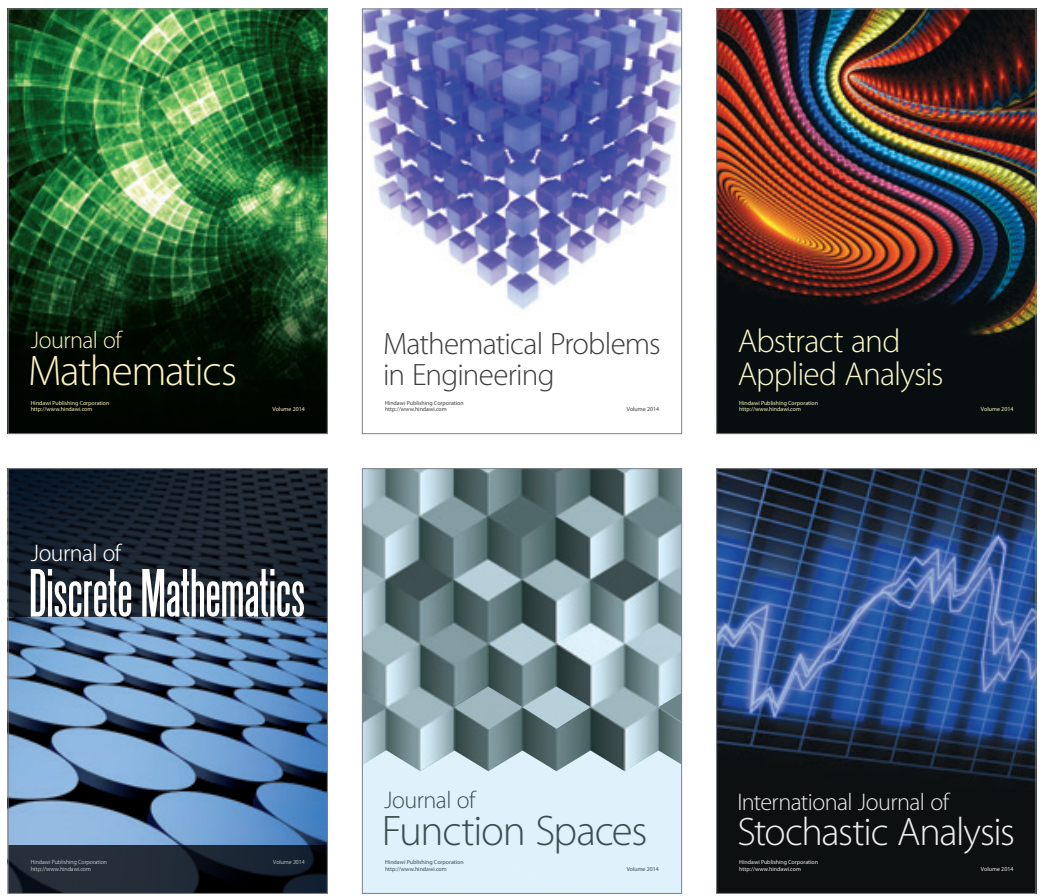

Journal of

Function Spaces

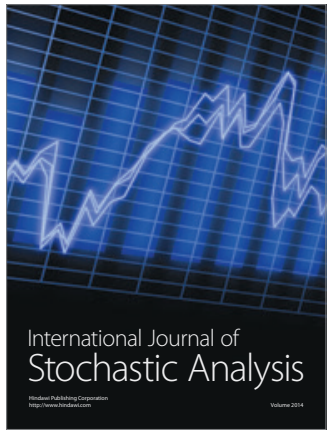

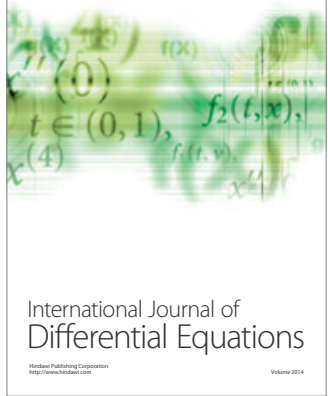
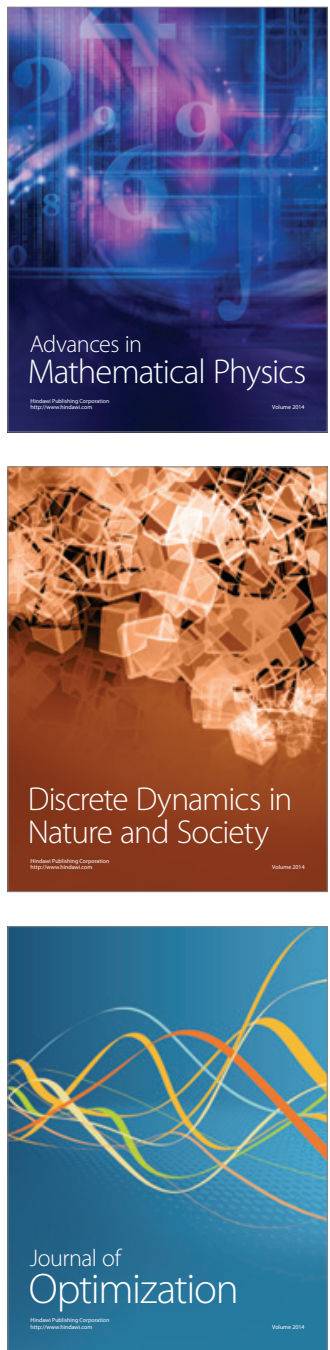